https://doi.org/10.7203/Normas.4.4686

\title{
DESCORTESÍA EN LAS PÁGINAS DE FACEBOOK DE FESTIVALES DE MÚSICA
}

\author{
IMPOLITENESS IN FACEBOOK MUSIC FESTIVALS PAGES
}

Luisa CHIERICHETTI

Università degli Studi di Bergamo

\section{RESUMEN:}

El presente artículo se centra en las interacciones que se desarrollan en Facebook (FB), el conocido sitio web de redes sociales que también es un recurso para la comunicación, y la promoción turística. Nos planteamos caracterizar este contexto sociocultural específico, que abarca comportamientos, actitudes y valores conocidos, aceptados y practicados en una comunidad discursiva, para luego describir el fenómeno de la descortesía en un corpus acotado de páginas de Facebook de festivales musicales, ofreciendo algunas reflexiones sobre sus características y sus funciones.

PALABRAS CLAVE: turismo musical, festivales musicales, Facebook, discurso digital, (des)cortesía.

\section{ABSTRACT:}

This article focuses on interaction in Facebook (FB), as one of the best known and effective social media networks for marketing in tourism communication and industry. After characterizing and describing the specific sociocultural context, which includes behaviors, attitudes and values as accepted and practiced in a discourse community, the article describes the phenomenon of impoliteness in a limited corpus of music festivals, offering some reflections on its features and functions.

KEY WORDS: musical tourism, music festival, Facebook, digital discourse, (im)politeness. 


\section{INTRODUCCIÓN}

La comunicación y la promoción turística de los últimos años se han desarrollado en gran parte en el ámbito de las redes sociales. En este trabajo nos centramos de manera específica en los festivales musicales en Facebook (FB). Nuestro punto de partida es la definición de sitios web de boyd ${ }^{1}$ y Ellison (2013), que los describen como una plataforma de comunicación en red en la que los participantes (amigos ${ }^{2}$ en FB):

- tienen un perfil único de identificación que se compone de contenido proporcionado por el usuario, contenido proporcionado por otros usuarios y/o datos proporcionados por el sistema;

- pueden articular públicamente conexiones que pueden ser vistas y enlazadas por los demás;

- pueden consumir, producir, y/o interactuar con secuencias de contenido generado por el usuario, proporcionadas por sus conexiones en el sitio (boyd y Ellison, 2013: 158) [traducción propia].

Fundado en febrero de 2004 y originariamente destinado a los estudiantes de la Universidad de Harvard, FB está abierto actualmente a cualquiera que tenga una cuenta de correo electrónico. Desde el lanzamiento de Facebook Ads, en noviembre de 2007, se ha convertido en una plataforma sobre la que terceros pueden desarrollar aplicaciones y hacer negocios a partir de la red social. Este programa está destinado a las empresas que quieran aprovechar las oportunidades ofrecidas por el contacto potencial con cientos de millones de internautas, creando una presencia distinta del perfil tradicional de usuario. Bajo los rótulos «Lugar o negocio local», «Empresa, organización o institución», «Marca o producto», «Artista, grupo de música o personaje público», «Entretenimiento»o «Causa o comunidad», las páginas permiten crear y mantenerse en contacto con los aficionados, contando con servicios personalizados basados en los sectores de mercado. Además, FB ofrece la posibilidad de crear «Social Ads», esto es, anuncios destinados a un grupo de usuarios con varias segmentaciones por intereses, contactos, edad, sexo y distribución geográfica.

La comunicación y la promoción turística se han beneficiado significativamente del importante desarrollo comercial de FB, que ha resultado especialmente rentable y útil para facilitar la interacción de los profesionales del sector con la comunidad de referencia, obteniendo datos sobre la satisfacción del cliente; por otro lado, se ha demostrado que ejerce una influencia positiva en las intenciones de comportamiento de los turistas (Grappi y Montanari, 2011). En los últimos años, los intercambios comunicativos entre turistas han llegado a ser el principal mensaje publicitario y turístico buscado por los consumidores-productores: la promoción del destino turístico

\footnotetext{
${ }^{1}$ La investigadora firma sus artículos sin iniciales mayúsculas, por razones personales y políticas que se describen en http://www.danah.org/name.html (23/8/2013).

${ }^{2}$ Escribimos con cursiva amigos cuando nos referimos a las conexiones en un sitio web de redes sociales, para diferenciarlo del concepto común de amistad.
} 
acaba siendo generada por los usuarios mismos, quienes hacen que la información circule y sea visible en la red de manera constante (González y Sanmartín, 2013: 252).

\section{El MACROGÉNERo PÁginA FB DE FeSTIVALES DE MúsiCA}

Al tomar en consideración la producción de discurso en la promoción del turismo musical en FB y su relación con el contexto sociocultural, nos planteamos situar este evento comunicativo dentro de una estrategia de búsqueda, posicionamiento $\mathrm{y}$ fidelización de clientes en un entorno turístico cada vez más extenso y competitivo.

Estas prácticas sociales y discursivas mediadas por ordenador pueden agruparse en dos de los bloques principales propuestos por Calvi (2010: 18-19), que sigue de cerca el modelo propuesto por García Izquierdo y Monzó Nebot (2003): el de la gestión, al comprender las interacciones entre operadores y público, y el de la descripción y promoción del destino turístico. Las páginas FB de festivales de música se configuran como un macrogénero, al ser productos que encierran una variedad de materiales muy amplia y por ser hibridaciones de secuencias textuales y de géneros informativos $\mathrm{y}$ persuasivos (básicamente guía práctica, anuncios publicitarios, folletos y páginas web), cuyo resultado final tiene un valor autónomo (Calvi, 2010, 2011). Este macrogénero funciona como un molde al que se adaptan, integrándose, los contenidos turísticos, bien sea en cuanto al patrón organizacional -extensión de los textos y de los aparatos multimedia e icónicos- bien sea en cuanto al enfoque funcional -mayor o menor relevancia de las funciones persuasiva, informativa, interactiva- (Calvi, 2011: 16).

El fenómeno comercial y turístico «festival», entendido como una celebración que se centra en un determinado tema y abierta al público durante un período de tiempo limitado, que puede convocarse anualmente o con menor frecuencia, y que incluye eventos aislados (Grappi y Montanari, 2011: 1129) es difícil de encajar en las categorías previstas por FB Ads; muchos festivales eligen como etiqueta «Lugar o negocio local», «Artista, grupo de música o personaje público», «Empresa, organización o institución» o también «Causa o comunidad». Los contenidos turísticos de las páginas FB van a integrarse, pues, en un género que no es propio del sector, amoldándose, no solo genéricamente, al canal de difusión (Calvi, 2010), sino a una plantilla estándar bastante rígida y definida, en la que las páginas comerciales están equiparadas con los perfiles personales (las referencias en el sitio son alternativamente páginas y perfiles) y los potenciales o actuales clientes siguen siendo amigos, por mucho que compartan una comunidad virtual planeada y construida por especialistas del sector. El inconveniente de la rigidez del medio, que se sitúa en las antípodas de la creatividad del sitio web, se compensa con un amplio beneficio desde el punto de vista del marketing en general y aún más desde una perspectiva turística, al permitir la interacción con la comunidad de referencia y, al mismo tiempo, proporcionar (por lo menos potencialmente) atención a los individuos. La interfaz de la archiconocida plantilla blanca y azul de FB despoja el mensaje comercial de su típica agresividad y «baja las defensas» del consumidor, pues inserta cognitivamente la operación comercial en un frame caracterizado como un ambiente en el que los individuos construyen su identidad -con su discurso, aportando 
materiales icónicos y multimedia-, sintiendo que están en un colectivo unido por un vínculo de solidaridad y aprecio (comunidad) ${ }^{3}$.

\section{MATERIALES, ENFOQUE METODOLÓGICO Y ORGANIZACIÓN DEL TRABAJO}

\subsection{Materiales}

El corpus tiene formato electrónico y se ha constituido reuniendo todo el material publicado en los muros (walls) ${ }^{4}$ de una selección de cuatro festivales desde la creación del perfil FB hasta diciembre de 20125: Sonorama (Aranda de Duero, Burgos), FIB (Festival Internacional de Benicasim, Castellón), Contempopránea (Alburquerque, Badajoz) y Aquasella (Arriondas, Asturias) ${ }^{6}$. Se trata de espectáculos que incluyen a autores, intérpretes y pinchadiscos de música pop, rock y electrónica, y que se celebran en lugares donde tradicionalmente no se llevan a cabo este tipo de eventos; se dirigen a un público mayoritariamente en etapa prefamiliar, que prefiere una receptividad y unos medios de transporte de bajo coste (acampadas, hostales, coches compartidos).

Se ha incluido todo el material de los muros para disponer de un contexto lo más extenso y rico posible, en el que se pudieran considerar las típicas actividades desarrolladas en FB con sus pautas recurrentes (aunque también hay otras áreas de discusión y de producción de discurso en FB relativamente menos utilizadas) y se ha organizado en un corpus electrónico de alrededor de 450000 tokens, que tiene la finalidad de ser representativo del uso lingüístico de un grupo específico de hablantes. El número de tokens, tal y como se presenta, es el resultado de una criba manual que ha permitido eliminar la muy alta repetición de palabras contenidas en la rígida plantilla de FB (las indicaciones de día, hora y a veces de lugar, los botones «Me gusta», «Responder», «Comentar», «Compartir», «A $x$ personas les gusta esto», «Ver comentarios anteriores», "Ver $x$ comentarios más», etc., que consideramos palabras funcionales vacías de significado. Los nombres de los participantes en el intercambio comunicativo se repiten cada vez que aparece una publicación (post); además, los posts de los amigos -y sobre todo los del Festival- pueden repetirse, ya que a lo largo del tiempo se va perdiendo la visibilidad de los mismos y, por lo tanto, la posibilidad de interacción con los demás usuarios; también hay notificaciones que se refieren a la actividad online de los participantes, como « $x$ ha actualizado su información» 0 « $x$ ha compartido un enlace». En el corpus, los contenidos están dispuestos de forma lineal y

\footnotetext{
${ }^{3}$ «When people use SNSs, they often identify friends, acquaintances, celebrities, and many others as Friends» (Parks, 2010 en boyd/Ellison, 2013: 155n).

${ }^{4}$ En la versión actual de FB este espacio individual de cada usuario donde aparecen sus actualizaciones y aquellas que son publicadas por sus amigos se conoce como se conoce como biografía (Timeline).

${ }^{5}$ Agradezco a Sabrina Baiguera la recopilación de la parte del corpus relativa al año 2012.

${ }^{6}$ Sonorama (https://www.facebook.com/sonorama.ribera) a partir del 30/6/2009, FIB a partir del 14/7/2011 (https://www.facebook.com/fibfestival?fref=ts), Contempopránea (https://www.facebook.com/ contempopranea?fref=ts) a partir del 11/3/2010, Aquasella (https://www.facebook.com/pages/AQUASELLA-FEST/19601087377 8362) a partir del 1/6/2011.
} 
en orden inverso ${ }^{7}$; es decir, los intercambios más recientes aparecen en la parte superior de la pantalla, y para ver las publicaciones más antiguas hay que desenrollar (scroll down) o seleccionar el enlace apropiado; en este último caso, la visualización «Ver comentarios anteriores», «Ver $x$ comentarios más» sigue el orden cronológico: arriba aparecen los más antiguos y abajo los más recientes. También hay casos de repetición masiva de posts que el Festival pide a cambio de la participación a un concurso, como el sorteo de entradas (por ejemplo, «Me gusta aquasella»o «Yo también voy a aquasella»).

Otro problema relacionado con la construcción y el análisis de este corpus reside en la gestión de errores ortotipográficos, ya que el lenguaje de los internautas escapa al control de cualquier tipo de autoridad normativa (Sanmartín Sáez, 2007: 52). Una parte de los errores -ortográficos, gramaticales, sintácticos- no es deliberada, pero otra parte, como la supresión de las tildes y de los signos de puntuación, es voluntaria y está estandarizada en intercambios de este tipo ${ }^{8}$ (por ejemplo, en el chat; véase Sanmartín Sáez, 2007, 52, 53). Es muy común simplificar la ortografía, utilizar abreviaturas (como $q, q e, t b$ por que, también) y suprimir sílabas (por ejemplo, na, pa por nada, para; en este caso, también reflejando por escrito unas formas coloquiales), porque se considera que son superfluas o que hacen más lento el proceso de la escritura rápida. Este fenómeno nos parece en aumento, según va extendiéndose el acceso a los servicios de redes sociales de forma cada vez más rápida a través de dispositivos móviles (teléfonos inteligentes, tabletas). A este proceso favorecido por los medios electrónicos se suma cierto relajamiento derivado de la proximidad entre los interlocutores -un rasgo coloquializador- y el deseo de ruptura ortográfica, típico de los usuarios más jóvenes (Sanmartín Sáez, 2007: 57). También hay que señalar que el intercambio en los muros se desarrolla casi exclusivamente en español, pero caben publicaciones en otras lenguas propias de España y en inglés ${ }^{9}$.

${ }^{7}$ La arquitectura de FB se halla en continua evolución; a partir de la primavera de 2013 el sistema de comentarios ha cambiado y ya no se corresponde exactamente con la realidad del corpus que describimos, que llega hasta diciembre de 2012. Los usuarios con un alto número de conexiones ven destacadas arriba, en la parte de «Comentarios» las conversaciones más activas y atractivas entre los lectores; también se ofrece la posibilidad de contestar de forma directa a las publicaciones hechas por otras personas para evitar que se junten varias respuestas y que se pierda el sentido de la conversación. Véase: https://www.facebook.com/notes/facebook-journalists /improving-conversations-on-facebookwith-replies/578890718789613 (23/8/2013).

${ }^{8}$ Díaz Pérez (2012: 316-317), sin referirse al especial tipo de lenguaje abreviado que muchos usuarios utilizan en la comunicación mediada por ordenador (CMO), llega a considerar que el error ortográfico, por su extensión, puede considerarse como un rasgo intrínseco de las redes sociales. Propone una clasificación en errores por descuido o tipográficos (ocasionados por la poca atención a la forma de lo escrito y por la publicación sin ninguna revisión); errores por defecto (la ausencia de tildes o de los signos de puntuación, por desconocimiento de las reglas básicas o como fruto de la rapidez); errores por desconocimiento (el emisor no domina el sistema lingüístico); errores conscientes (por ejemplo para imitar el habla de un personaje).

${ }^{9}$ Señalamos que las comunicaciones oficiales del FIB, que tiene un amplio público anglosajón, siempre van en español e inglés. 


\subsection{Enfoque metodológico}

Nos planteamos considerar el evento de comunicación y de interacción que llamamos discurso como uno de los elementos de las prácticas sociales que influyen en la sociedad y caracterizan a la modernidad tardía, a la que al mismo tiempo vertebran y construyen. Dentro de esta perspectiva, nos centramos en los cambios culturales creados por los avances en las tecnologías de la información, fijándonos en las nuevas posibilidades de relaciones interpersonales (Chouliaraki y Fairclough, 1999: 2, 3) que se dan en FB, en la significatividad y el interés de las dinámicas socio-técnicas que han llevado a millones de personas a utilizar la tecnología para colaborar, compartir información y socializar (boyd y Ellison, 2013: 160). Entendemos FB como esfera pública, constituida por una manera especial de uso del lenguaje de un espacio público (véase Chouliaraki y Fairclough, 1999); los participantes en la interacción nunca son unipersonales, sino que se dirigen a grupos sociales y encarnan ellos mismos grupos sociales, actuando a veces como instancia productora y otras como instancia mediadora entre la sociedad y el público (Charaudeau, 2009: 261-262).

FB es una práctica sumamente compleja en su forma, en las relaciones sociales de su producción y en la red de prácticas en la que se inserta, se desarrolla a través de grandes distancias espaciales y temporales y depende de sofisticadas tecnologías de mediación (Chouliaraki y Fairclough, 1999: 41-42). Dentro de esta práctica, el uso comercial de sitios web de redes sociales es un cambio económico, social y cultural que existe como discurso y, al mismo tiempo, como una serie de procesos que se realizan fuera del discurso (Chouliaraki y Fairclough, 1999: 4).

Por otro lado, situamos nuestro análisis en el ámbito de los estudios sobre el fenómeno de la (des) $\operatorname{cortesía}^{10}$, que en las últimas décadas han revisado críticamente las teorías iniciadoras de Lakoff (1973), Brown y Levinson (1987) y Leech (1983), planteando una perspectiva basada en el género para explicar la variación cultural y contextual de este fenómeno (Garcés-Conejos Blitvich, 2010) ${ }^{11}$. Nuestra contribución se propone describir algunos recursos lingüísticos de la (des)cortesía en el macrogénero Página FB de Festivales de Música, entendido como un contexto sociocultural específico, que abarca comportamientos, actitudes y valores que son conocidos, aceptados y practicados en una comunidad de hablantes (Bravo, 2004b: 24-31; Hernández Flores, 2004: 96-97).

${ }^{10}$ Las reflexiones en torno a la (des)cortesía entendida como un fenómeno que se desprende de la contextualización del objeto de estudio están llevando a abandonar la tradicional dicotomía entre cortesía y descortesía, que se tienden a considerar como un continuum. De aquí que utilicemos en este trabajo el término consolidado (des)cortesía para reflejar la representación conjunta de ambos conceptos (Brenes Peña, 2007: 207), reservando descortesía, sin paréntesis, para el polo del continuum del que nos ocupamos más concretamente.

${ }^{11}$ No nos detenemos en una revisión detallada de las teorías sobre (des)cortesía. Para una síntesis eficaz del estado de la cuestión sobre los estudios de la (des)cortesía remitimos a Bernal (2007: 57-89), Díaz Pérez (2012: 45-87) y a Landone (2009: 15-73). 


\subsection{Organización del trabajo}

El producto de comunicación que vamos a analizar es el resultado del empleo de la lengua en el terreno de la promoción turística en un contexto de contenidos en su mayor parte generados por los usuarios. Se trata, pues, de un discurso de especialidad que se ubica en la perspectiva de la publicidad espontánea -o inconsciente- generada por los usuarios/consumidores (Nicolás Ojeda, 2013).

Dentro del contexto situacional y sociocultural en el que se enmarca la situación concreta de nuestro corpus de páginas FB vamos a individuar unidades discursivas que puedan considerarse como estrategias comunicativas voluntariamente encaminadas a atacar la imagen del otro, creando conflicto social y desarmonía (Culpeper et al., 2003: 1546). Vamos a incluir también, cuando sea posible, la percepción o la evaluación por parte del destinatario, ya que «la cortesía o la descortesía de un acto de habla depende en muchas ocasiones de la interpretación que de éste hacen los interlocutores» (Briz Gómez, 2001: 158).

\section{ANÁLISIS}

El discurso de los festivales de música en FB se construye y desarrolla de manera textualmente híbrida, y se configura como un ejemplo significativo de promoción turística polifónica que incluye también las voces potencialmente conflictivas e irreverentes de las personas que interactúan en FB. Los usuarios desarrollan una actividad en línea socialmente significativa que deja huellas textuales (Herring, 2004), escritas y multimedia, grabadas de forma permanente, también con gran distancia espacio-temporal.

El grupo FB de un festival se sitúa en un entorno concebido para optimizar la interacción de tipo comunitario y gestionado con la finalidad de lograr la rentabilización del uso de la red social. La figura profesional que se identifica con el festival es el gestor (community manager, CM) o emisor de las publicaciones del muro de FB que propone temas de discusión, crea eventos, mantiene el contacto con los usuarios y básicamente deja fluir la discusión entre ellos; estos, por su parte, pueden escribir sus propias publicaciones (las páginas son accesibles a todo el público, mientras que para interactuar hay que identificarse como usuario de una cuenta FB), declarar su aprecio (con el botón «Me gusta»), compartir la publicación con otros amigos o comentarla. Los amigos del festival se configuran como una comunidad virtual de personas que no necesariamente se conocen fuera de línea, pero que posiblemente se encuentren o se hayan encontrado físicamente en el evento, aunque sin llegar a conocerse personalmente; están unidos por el interés cultural hacia cierto tipo de música y por el interés turístico de querer asistir o de asistir al festival. Este distanciamiento espaciotemporal en el que se mezclan intimidad e impersonalidad hace de FB un sistema abstracto absolutamente «moderno» (véase Giddens, 1994).

En el discurso del festival en FB se destacan las tres funciones principales de los géneros turísticos señaladas por Calvi (2011: 35): informar, instruir y persuadir. En FB 
la empresa turística genera vínculos de afinidad, estrecha relaciones personalizadas, ofrece información en tiempo real, soluciona conflictos de forma inmediata, etc. Al estimular la participación generando contenidos de interés común, la producción discursiva también sirve como anclaje de los materiales multimedia (sobre todo videoclips, pero también fotos), con enlaces web que amplían intertextualmente, de forma potencialmente infinita, los límites de la red social. El gestor no proporciona siempre textos elaborados específicamente para FB, sino que se propone como receptor pasivo de la información y aglutina información extraída de distintas fuentes que, mayoritariamente, se encuentran en la web ${ }^{12}$.

La comunidad del festival comparte un interés turístico fuera de línea, el de participar en este tipo de eventos musicales y, de manera especial, en un festival en concreto $^{13}$. Esta pertenencia al grupo está marcada por la construcción de una identidad comunitaria otorgada y reconocida por la organización del festival: los seguidores de FIB son fibers o fiberos, los de Sonorama sonoritos o sonorit@s, los de Aquasella aquasellinos, los de Contempopránea contempopráneos.

Reconocemos en los intercambios comunicativos de FB una interacción conversacional de tipo periférico, ya que se trata de un diálogo entre personas virtualmente (que no físicamente) presentes que mantienen una relación idealmente simétrica en cuanto a sus derechos a participar en el intercambio de contribuciones comunicativas (Bravo, 2004a: 6), en el que la toma y cesión de turnos no es predeterminada, ni es negociada de forma previa (Briz Gómez, 2000: 11). Las instancias enunciativas que observamos se mantienen en los dos ejes festival-amigos y amigosamigos, y se articulan en secuencias aserción/réplica. Al receptor directo del enunciado emitido se le suma un segundo receptor masivo que tiene la posibilidad de unirse a la interacción, ya que la comunicación en Internet y en general en los medios de comunicación de masas siempre está presente un receptor polifónico intrínseco (Kerbrat-Orecchioni, 1986: 34; Díaz Pérez, 2012: 34). Podemos afirmar, pues, que la interacción no es diádica e interpersonal, sino que se da en una situación polilogal (Kerbrat-Orecchioni, 2004), intergrupal y mediatizada, que se fundamenta en la identidad social y no en la individual (Garcés-Conejos Blitvich, 2013: 170).

El marco de FB condiciona el funcionamiento de los mecanismos de realización textual en el proceso de recepción/interpretación de los textos, ya que la identificación del «medio» por parte del receptor por un lado construye una credibilidad comunicativa y por otro provoca, por el fenómeno de la intertextualidad, ciertas expectativas comunicativas (Bustos Tovar, 1999: 108) que alcanzan varios aspectos como el objetivo interlocutivo perseguido por los participantes, las relaciones sociales existentes entre

${ }^{12}$ La información se distribuye, se comparte y, a menudo, se repite en la Red a través no solo de FB, sino también, por ejemplo, de Twitter, Tuenti, Flickr, YouTube, Spotify, Tumblr, Instagram, etc.: «... the technical affordances that define a social network site have become increasingly fluid. Of course, people's practices, expectations, and social norms have also co-evolved alongside the technical features and social interaction opportunities» (boyd/Ellison, 2013: 152).

${ }^{13}$ Naturalmente, también comparte un interés cultural por la música, que se refleja como discurso de especialidad en las páginas FB; sin embargo, en este trabajo no vamos a ocuparnos de esta vertiente discursiva. 
ellos, las normas específicas que regulan la interacción y el comportamiento comunicativo que se considera adecuado (Brenes Peña, 2011: 39).

En la aplicación comercial de FB, la identidad de los sujetos está formada heterogéneamente por efecto de los distintos posicionamientos del usuario (Chouliaraki y Fairclough, 1999: 25), que es, a la vez, amigo y cliente. Vamos a comprobar, pues, cómo el discurso de la promoción e información turística se hibrida con la interacción típica de una relación vivencial virtual de proximidad (Briz Gómez, 2001: 41), generando unas dinámicas interactivas sustentadas en la cercanía interpersonal, el contenido relacionado con las vivencias concretas y el interés turístico que se comparte, en el que descuellan las actividades en el ámbito de la (des)cortesía. Por un lado, no faltan ejemplos de actos de refuerzo de la imagen tales como halagos, cumplidos, alabanzas y agradecimientos, que valoran, reconocen o aprueban algún aspecto de la experiencia turística (Abelda Marco, 2003: 300), como podemos comprobar en los intercambios del tipo de $(1)^{14}$ :

(1) Carmen Delgado Ha sido mi primer Sonorama... el de día es toda una gran fiesta!! Pedro Benavides Villarragut Para otro año alli estaremos......ya queda menos.

Carmen Solans cada año mejor!!

Wismansson AC su bli me

Gabi Montes ESPECTACULAR! GRACIAS POR TODO

Javier Hueso Gran festival!! Si me cortasen ahora mismo me saldría alcohol en vez de sangre... Buena música, mejor gente, ambiente inmejorable!

Ane Straat ESPECTACULAR! Deberías montar un sonorama 14.5 durante el invierno!! DE VERDAD!!!!!!!

Tatiana Restrepo Se ha terminado por este año. El año que viene mas. Me ha encantado. Felicidades por la iniciativa

Patricia Muro Gracias a vosotr@s!! Ha sido nuestra «primera vez»y repetiremos fijo!!

Vanessa Dominguez Merino ha sido nuestra primera vez en Sonorama y todo ha sido perfecto. Buena gente y buena música!!!!!

Alex Fabres Calavia una gran experiéncia que me gustaría repetir...Y LOVE SONORAMA!!!

Romi Sique Mola Vuestro festival ya es el mejor!! Ha sido increible otra vez. Larga vida! Gracias, gracias, gracias!

Quike Garcia han sido unos dias magicos!! gracias aranda! gracias sonorama! nos vemos pronto!

En los actos de cortesía valorizante de (1) se destaca una alta frecuencia de adjetivos calificativos y de superlativos, ya ampliamente descrita en los estudios sobre la lengua del turismo (Calvi, 2006). Sin embargo, nos interesa destacar en este corpus un tipo de interacción que se sitúa en el polo opuesto del continuum de la (des)cortesía, es decir, la presencia de una variedad de fenómenos descorteses cuya intención es ofender deliberadamente (Culpeper, 2005: 39). Adoptamos como punto de referencia la

14 A la hora de reproducir los fragmentos extraídos de FB mantenemos la grafía original, utilizando el subrayado para marcar el énfasis propio. 
definición de descortesía proporcionada por Culpeper (2005: 38), que abarca, por un lado, la intencionalidad del emisor, y por otro, la interpretación del destinatario:

Impoliteness comes about when: (1) the speaker communicates face-attack intentionally, or (2) the hearer perceives and/or constructs behavior as intentionally face attacking, or a combination of (1) and (2) ${ }^{15}$.

Como podremos comprobar, la presencia de actos descorteses tiene efectos paradójicos por el conflicto entre la opinión del cliente, expresada de forma directa y a veces brutal u ofensiva, y el discurso de la promoción turística ${ }^{16}$.

Señalamos la presencia en nuestro corpus de fenómenos de descortesía aparente (banter o mock impoliteness) que no ataca la imagen del interlocutor, diferenciada por Culpeper (2005: 37) de la descortesía auténtica (genuine impoliteness). Se trata más bien de fenómenos de anticortesía (Zimmerman, 2003) que refuerzan el sentimiento de pertenencia al grupo y, lejos de ser una muestra de hostilidad, llegan a provocar cierto efecto de cortesía. Como ejemplo del uso del insulto como función de la identidad colectiva de los miembros del grupo y de las relaciones intergrupales (Upadhyay, 2010), aportamos el intercambio (2), en el que el apodo denigrante cabrón no se utiliza con intención de amenaza, sino como vocativo amistoso; de hecho, el afectado no expresa ningún tipo de queja ni de reacción negativa, sino todo lo contrario (véase también Brenes Peña 2007: 203):

(2) Jaime Brodie Es facil viernes carlsberg y sabado barcelo!!

Forma Way jajajaja no flipes jaime tan facil no es

Jaime Brodie lo tengo muy claro man jajaj alli nos vemos cabron

Forma Way con maceo plex seguro que si que nos vemos en la carlsberg

Forma Way y yo te kiero mostrar al dr tio

Jaime Brodie ok te are caso pero tu tienes k ver a kaden como sea el viernes!! dinamitaaaa

Forma Way si a pasado por awakenings caña ok trato echo

La «conversacionalización» del discurso turístico lleva a la pantalla del equipo electrónico la inmediatez, la espontaneidad, la informalidad y el uso de la descortesía no solo en situaciones en que hay voluntad clara de agresión, sino también en situaciones de familiaridad, o cuando el consenso social (en este caso relacionado con los jóvenes) comparte una idea de lo que es más o menos ofensivo (Calsamiglia y Tusón, 1999: 163; 172).

También comprobamos que se dan intercambios propiamente descorteses entre amigos, como el ejemplo (3), en el que el desacuerdo atañe a la escasa inteligibilidad

15 Bernal (2007: 86) critica esta definición argumentando que si el interlocutor no reconoce el ataque no existe efecto descortés. Sin embargo, en nuestro corpus, como veremos, las reacciones del Festival no se corresponden exactamente con las evidentes amenazas de la imagen que recibe; de ahí que la definición original de Culpeper nos parezca más adecuada.

${ }^{16}$ Para una visión más completa de las dinámicas de interacción en este corpus, véase Chierichetti (2012) y (2013). 
del mensaje, situándose en el plano metalingüístico. La usuaria ofendida reacciona amenazando a su vez la imagen de la amiga -atacando su apariencia física, sobre la base de la fotografía que aparece en el perfil de FB- y defendiendo la imagen social determinada por su rol específico de aquasellina («tengo derecho a escuchar la musica qe me gusta sin meterme con nadie!!!»); también nos proporciona una interesante visión de la finalidad del grupo: dar opiniones.

(3) Arancha Rodriguez Perez cada uno va como le sale de los cojones yo nodigo ni qe pongan una cosa ni otra si no qe ya qe hay 3 zonas de musica qe pongan para todos los gustos y ya esta.... a unos les gusta el schranz pues pa una carpa a otros el electro pues pa otra y ya esta......y el open air mas grande y abierto y dejaros ya de tanta zapatilla y tanta alpargata a cada uno le gusta la música qe le sale del cono y listo era lo qe faltaba $\mathrm{xd}$ !

Marta Liébana Auqué Joder, escribes con el puto culo tronca, asi no se te puede tomar en serio!

Marta Liébana Auqué Lee un libro y dejate de tanto schranz

[...]

Arancha Rodriguez Perez y la penca qe dice qe escribo como el culo y qe me lea un libro mira maja ya qisieras tener los estudios y la cultura qe yo tengo qe no te llega ni al tacon de mi zapato y si yo me tengo qe leer un libro tu pasate $\mathrm{x}$ la pelu $\mathrm{y}$ cambiate ese pelo qe aqui estamos pa dar opiniones no pa meternos con la peña y faltar qe yo pago entrada como los demas y tengo derecho a escuchar la musica qe me gusta sin meterme con nadie!!!

Como acabamos de ejemplificar, en nuestro corpus podemos hallar varios aspectos interactivos que se sitúan en el ámbito de la (des)cortesía. Sin embargo, nos vamos a centrar en un fenómeno que nos parece muy interesante desde el punto de vista de la comunicación turística, es decir, las actividades ofensivas de ataque a la imagen del festival, ya que, por mucho que los mensajes provocativos sean un fenómeno típico de las interacciones mediadas por ordenador, nunca hay que olvidar que estamos hablando de un uso comercial de la red.

En el ámbito de la comunicación turística y de la interacción intergrupal entre los festivales y los usuarios, llama la atención el uso masivo de actos expresivos de crítica que se sitúan en la descortesía sin ningún tipo de atenuación y con el evidente objetivo de amenazar la imagen del destinatario, descortesía descarnada (bald on record impoliteness), según la clasificación de Culpeper (1996 y luego 2005: 41). A continuación presentamos algunos posts en los que el ataque descortés se dirige al vosotros que representa a los que trabajan en el festival cuando hay cambios en el cartel -en los ejemplos de (4) a (10) - o fallos en la organización del evento turístico -en los ejemplos de (11) a (13)-:

(4) Jorge Gregorio Sanz no teneis ni puta idea de organizar un festival,sois unos estafadores y unos ladrones,que defraude de festival

(5) Joyu Waitforit Ross Pero qué puto asco dais. No habéis encontrado mejor forma de vender abonos y dar de qué hablar no? Teníais que pegarle esta hostia en la cara a vuestro público habitual. Bravo. Que desgraciados sois. 
(6) Nacho Cenitagoya No se por que abris este debate, si despues quitais las opiniones de la gente. ESTO ES LAMNTABLE, SOIS BASURA.

(7) Fá Cil (Fat Bad Boy Lee) ¡PEGAOS FUEGO!

Kafka Sabadell Sois la peste. De verdad!

(8) Jorge Freire Este FIB es una puta mierda.

(9) Fernando Ibáñez Moscardó Vaya basura de cartel

(10) Laura Descalabro patético!

(11) José Angel Benavente Soba la mejor actuacion fue la de los de seguridad ke permitían colarse a gente al festival a costa de romper toldos(ke todos vimos al de naranja y como su superior no le decia nada....), ademas de robar entre tiendas y coches y cuandose lo dices, nos contestan ke no hay ley dentro del recinto..... menuda organizacion de los cojones, a mi no me toco pero sino pa vosotros teneis.... porke creo recordar ke indicabais que teniais seguridad y yo no vi a nadie una vergüenza de organizacion....

(12) Jexus Sanjuan Esto se avisa antes k mucha gente ya habia reservado en casas. En diciembre en arriondas.com ya salio la informacion de que el festival cambiaba de fin de semana segun los promotores, pero no habeis dicho nada en 2 meses y encima reducis un dia?? Estas $\mathrm{k} t$ pegas el viaje desde madrid pa un sabdo mas...... Aki teneis el enlace de hace 2 meses http://www.arriondas.com/2011/12/aquasellacambia-defecha/ CABRONES!!!!

(13) Eduardo Navarro Zarraga PERO ESTAMOS LOCOS O K??? EL SELLA ES FINDE DE PIRAGUAS JODES NO JODAMOS LA MARRANA HOMBRE OS AKABAREIS CARGANDO EL FESTIVAL VERÉIS

Más raramente se amenaza de manera directa la imagen del gestor de la página, como en (14). En (15), los actos amenazantes que dos amigos cometen hacia el CM se les añaden, a posteriori, sendos actos de cortesía reparadora que compensan retrospectivamente al destinatario (Bernal, 2007: 119):

(14) Jordi Elesgaray El administrador esta tan jodido que dudo que haga acto de presencia, me comentan tambien algo de Carlos Jean? ojjojojojojojoojojojjoojjooj

(15) Diego Ciller Yo lo había posteado antes pero lo he borrao por respeto al CM xq no tiene culpa, pero que se lo miren xq ha sido patético. De música ni hablo. El cartel del sos es mejor por ahora y relación calidad/precio, ni hablamos

Fernando Villar ok. Lo elimino por respeto al CM. Quién es el CM?

Diego Ciller Ni puta idea pero vamos un mono tardaría menos en hacer copy paste. jajaj seguro que nos lees, no te enfades q es broma! si eres una chica te invito a unas fantas en el fib.

Fernando Villar CM es mujer o ciber?

Fernando Villar Es bromaa... Yo te invito a unas mirindas

También comprobamos cómo el insulto se puede dirigir directamente a los artistas que van a actuar en el festival. Es el caso de la inclusión del músico David Guetta en cartel del FIB de 2012, que provocó una avalancha de ataques de este tenor: 
(16) Daniel Mozota Loeches QUE COJONES PINTA EL GUETTA DE LOS COJONES EN UN FESTIVAL DE ESTOS........PERO NO OS DAIS CUENTA DE QUE ESE HOMBRE NO TIENE NI PUTA IDEA DE PINCHAR.........NADA MAS QUE HACEIS ES LLENNARLE LOS BOLSILLOS DE EUROS.....CON LOS PEDAZOS DE DJ'S QUE HAY Y ME TRAEIS A ESTE PINTAMONAS...POR LO DEMAS MUY BIEN EL CARTEL...SIEMPRE TIENE QUE HABER UN GARBANZO NEGRO EN EL COCIDO JODER.......

(17) Hrundi V. Bakshi Venga, aceptamos David Guetta como animal de compañia (pero no como músico ni como independiente..)

(18) Pedro Peñas Robles despues del monegros 2011, asco da !! estos festivales han perdido lo esencial. A tomar por culo el Guetta ese!

El relativo anonimato de los usuarios ${ }^{17}$, que en $\mathrm{FB}$ se da menos que en otros tipos de conversaciones tecnológicas, la distancia espacio-temporal y la ausencia de factores contextuales (como el género, la edad o la orientación sexual) favorecen una mayor libertad expresiva y, por lo tanto, este fenómeno discursivo que causa daños a la imagen del festival al mismo tiempo que refuerza la imagen positiva del emisor.

Por un lado, los mensajes deliberadamente hostiles, provocativos o pensados especialmente para generar respuestas insultantes, son un fenómeno típico de la CMO. Pero no hay que olvidar que, dentro de un uso comercial de la red, por mucho que se le provoque, el gestor de un servicio (turístico) difícilmente puede reaccionar a las provocaciones de los clientes, aunque puede borrar algunos de los comentarios -hecho que los usuarios critican contundentemente, como comprobamos en el ejemplo (6)-. Por esta razón, los posts reactivos no se corresponden cuantitativamente con los de los usuarios; la respuesta más frecuente es el silencio, aunque no podemos efectivamente saber si el gestor se ha comunicado con los usuarios a través de mensajes privados. De ahí que resulte difícil atestar qué tipo de efecto tienen en el destinatario estos actos amenazadores de la imagen (Culpeper et al., 2003: 1562). Entre las escasas estrategias reactivas, en (19) notamos que se intenta restablecer la autoimagen, tildando de «integristas» a los usuarios y destacando la presencia de saboteadores profesionales (trolls y haters). En (20), el festival adopta estrategias reactivas distintas en el mismo post, dirigiéndose a dos usuarios: en el primer caso, contraataca poniendo en entredicho la competencia del amigo en el ámbito musical y, por lo tanto, amenaza su imagen de rol; en el segundo, aparenta no percatarse de la implicatura y utiliza una cortesía estratégica para reparar la amenaza recibida y reestablecer el equilibrio.

(19) FIB vemos que sigue existiendo el mismo integrismo que el año pasado con Julieta Venegas (o antes con The Prodigy, o con el mismísimo BRIAN WILSON, e incluso LEONARD COHEN -sin llegar a estos niveles, cierto-). vemos que

${ }^{17}$ Los usuarios de Facebook se comprometen a proporcionar sus nombres e información reales y un nombre de usuario que la gestión de FB puede eliminar o reclamar si lo considera oportuno (http://www.facebook.com/legal/terms); por mucho que sea posible simular identidades o mantener el anonimato, la ocultación de la identidad y la asunción de roles ficticios son mucho menos frecuentes y no son consustanciales a FB con respecto a otros tipos de conversaciones tecnológicas, como el chat (cfr. Sanmartín Sáez, 2007: 12). 
muchos caen en los tópicos que han escuchado en algún sitio. vemos que muchos creen saber cómo se organiza un festival, y que tienen incluso más datos que nosotros sobre lo que pagamos a los grupos. vemos que como en cualquier foro o red social, la gente cree que la existencia de trolls + haters + los que están disgustados hacen pensar que representan a la gran mayoría (cuando aquí hay más de 73.000 seguidores). y no, no estamos buscando otro público, simplemente, no pueden gustarle los mismos 100 grupos a 50.000 personas distintas.

(20) Diego Romero Muñoz Y el viernes puta basura.

Adrian de la Rosa o yo no tengo ni idea de música o estos carteles no valen ná.... FIB Diego Romero Muñoz, solo alguien que despecia a Bob Dylan (entre tantos otros) podría utlizar esas palabras. Peor para ti, de verdad.

Adrian de la Rosa, si no conoces a los grupos y te apetece, puedes escucharlos en nuestras listas de spotify (http://open.spotify.com/user/fiberfib) o verlos en las de youtube (http://www.youtube.com/fiberfib);)

(21) Ronald Marcelo Cerna Ahora bien, me parece una reverenda estupidez lo que habeis hecho este año de poneros de acuerdo con el Optimus y el BBk para poner las mismas fechas de festival. EL año pasado tuve la oportunidad de ir a ambos, BBk y FIB, y este año pensaba hacer lo mismo, y al igual que yo mucha gente. A ver si para el año que viene manejan mejor las cosas, que absurdo todo.

FIB Ronald Marcelo Cerna, ¿siempre tenemos la culpa de todo? Nosotros mantenemos nuestras fechas, son los otros festivales los que se han movido. Nosotros no nos hemos puesto de acuerdo con nadie, entre otras cosas porque no ha habido ninguna consulta. A los que más perjudica es a vosotros, eso está claro, porque no se puede estar en dos sitios a la vez. Sí que es absurdo, sí.

(22) Nera R. Valls ¿Dónde se pueden dejar las bicis? ¿En la entrada del festival? me refiero a si se pueden aparcar allí mismo para no tener que caminar tanto...

Rafa Simó Sancho El año pasado había «aparcabicis» en las entradas de los campings y delante de la entrada del festival

Nera R. Valls gracias Rafa, si no fuera por la gente no nos enteraríamos de nada, pues $\ll \mathrm{FiB} »$ nunca contesta

FIB Hola Nera R. Valls, estamos respondiendo a todos los mensajes que se nos envía. Con lo cual lo que dices no es verdad. Habrá espacio para que puedas hacerlo. ¡Saludos!

El ejemplo (22) nos da pie para constatar no solo la reacción defensiva del festival, sino también cómo el acto descortés dirigido hacia un destinatario que está fuera del grupo (aunque pueda en cualquier momento unirse a la interacción) refuerza los lazos y el sentimiento de colaboración entre usuarios, fortaleciendo los vínculos identitarios del grupo, contrapuesto con la organización misma. De hecho, uno de los «efectos secundarios» más llamativos de la descortesía es la intensificación de los lazos de afiliación entre amigos. Por un lado, con el escarnio de la organización del festival, el grupo se autoafirma agresivamente; por otro, el usuario argumenta en nombre del grupo a la vez que se destaca dentro del mismo (Ilie, 2001: 259) y no ataca en función únicamente de sus creencias personales, sino que asume las del grupo al que representa. Asimismo, no ataca solamente las actuaciones de un destinatario individual, sino las de todo el grupo en el que este se incluye (Díaz Pérez, 2012: 109). 
Un lugar privilegiado en el que se puede plenamente apreciar el respaldo que se dispensan los participantes del grupo se da cuando la descortesía se basa en unas estrategias indirectas (descortesía encubierta u off-record impoliteness, según Culpeper, 2005: 44) mediante implicaturas, en un juego sarcástico, irónico o burlesco. En (23), la ironía nace de la repetición ecoica de unas proposiciones escondidas en el subtexto; los amigos imitan los posts que anuncian las confirmaciones de los artistas, o los que piden no nombrar la competencia en las páginas oficiales de un festival. Este procedimiento se enlaza con la creación irónica de un nuevo festival de amigos voluntariamente exiliados del festival original, por divergencias musicales, y el juego de parónimos con el nombre de un festival evidentemente poco apreciado por los usuarios (Arenal Sound/Orinal Sound):

(23) Juanfran Bautista Pardo NUEVA CONFIRMACION: Cabeza Casco DJ Cabeza Casco Antes pincho en el Orinal Sound Juanfran Bautista Pardo JAJAJAJJAJAJA EXILIADOS FESTIVAL FOR EVER!

Cabeza Casco Calla, q tu ya has pagado el bungalow y tiene q ir por cojones Juanfran Bautista Pardo TOPO 0 - CAGCO 1

Cabeza Casco Este el el Facebook del fib, para hablar del exiliados vayan a su facebook correspondiente, gracias

En las estrategias descorteses encubiertas el emisor pretende, en cierto modo, enmascarar o disimular su verdadera intención, con el fin de evitar que le sea atribuida la responsabilidad de haber realizado un acto amenazador (Escandell, 2006: 158). Sin embargo, las estrategias indirectas no son menos descorteses que las directas, sino todo lo contrario: pueden ser más ofensivas (Culpeper, 2005: 44). El esfuerzo intelectual que supone la interpretación correcta del post, fundado en el desfase entre enunciado y enunciación, provoca un efecto local perlocutorio de complicidad y, por ende, refuerza el sentimiento de pertenecer a una comunidad discursiva. En este sentido, la descortesía indirecta comparte el mecanismo en el que se basa el humor, o, invirtiendo los términos, el humor a menudo incluye la descortesía (Culpeper, 2005: 46). En la descortesía de tipo encubierto es aún más evidente la necesidad de tener en cuenta el contexto situacional y sociocultural, que llega a ser imprescindible para la correcta interpretación de (24); también las amenazas presentes en la pregunta de (25) y la afirmación de (26) son bastante oscuras, pero se explicitan a continuación:

(24) Kanelaitis Dc Alguien quiere un abono?

(25) Cabeza Casco Hola ¿Este año vale la pulsera del Fib 2011 para acceder al recinto? Como vienen los mismos...

(26) David Smith Sois como Apple. Sacan un modelo iMac nuevo antes de Navidad y cuando vas a comprarlo ni lo tienen en stock ni tienen ni idea de cuando lo vayan a tener. Así, que acabas cambiando de marca...

(27) Yoyi ComoMolo jajajaja Peazo cartel, eh??? Ya podéis prolongar el plazo, ya!! jajajaja Os habéis vuelto locos para confeccionarlo, eh??? Hay zona de tercera edad en el área vip???? JAJAJAJAJA 
(28) Javier Gordillo y encima os han hackeado la web! aunque seguro que con el nivel de actualización que lleváis no os habréis dado ni cuenta...

(29) Jorge Albargues vendo entradas para el fib o mejor, cambio entradas del fib por entradas para el primavera sound, imagino que nadie aceptara el trueque...

Fernando Villar Jajaja...ni de coña Jorge...Aunque, móntate en algún Bus Turístico y puede que pilles a algún guiri despistao...Suerte!

Entre las estrategias indirectas recordamos las secuencias suspendidas, que tienen un alto valor comunicativo e intencional: el hablante suspende deliberadamente el enunciado cuando supone que el oyente tiene información extralingüística, lingüística y contextual suficiente para reconstruir el significado a través de implicaturas (Herrero, 1996: 125-126). En los ejemplos de (30) a (33) también apreciamos cómo los atenuadores, cuando se insertan en una interacción abiertamente polémica, más que suavizar la agresividad verbal otorgan un matiz irónico-despectivo:

(30) Carlos Arróniz Cagadita hacer coincidir a Lori Meyers y Sidonie... (ahí lo dejo)

(31) Marta Font Quien cono va a ir al concierto de julieta venegas en el FIB? que penita...

(32) Carlos Mos q asquito de cartel....madre mia

(33) Jorge Medina Pérez seguid asi q vais bien...que penita. espero que sorprendais!

El ejemplo (33) también nos da pie para comentar cómo los enunciados corteses (halagos, cumplidos, alabanzas, agradecimientos, etc.) también pueden utilizarse con finalidad descortés, mientras que los siguientes, (34) y (35), nos permiten observar cómo las estrategias directas e indirectas (cumplidos, agradecimientos y deseos evidentemente irónicos) con frecuencia aparecen mezcladas (Culpeper, 2005: 42):

(34) Jose Ignacio Murciano si ya el año pasao la cagasteis con el cartel este año os habeis caido con todo el ekipo tenia dudas si ir este año o no pero viendo lo visto no voy fijo y conmigo habra muxa gente $\mathrm{k}$ piense igual......al final vais a conseguir $\mathrm{k}$ aquasella se deje de celebrar un BRAVOOOOO $\mathrm{x}$ esa organizacion tan buena $\mathrm{k}$ teneis

(35) Gonzo Mahouteista Pues lo unico que puedo decir es, GRACIAS POR JODERME LAS VACACIONES. Tenia reservado hotel para la semana del descenso y depues bajarme al creamfields(ya tengo las entradas) con lo cual espero ke os vaya bonito.

Finalmente, observamos cómo un aspecto importante del uso de estrategias descorteses se fundamenta en la presencia de un receptor polifónico intrínseco, a la que aludimos al comienzo de nuestro análisis. Aunque sin duda menos que en otros géneros, como por ejemplo en la tertulia televisiva (estudiada en Brenes Peña, 2011), la dimensión pública propia de FB favorece la intensificación de los rasgos relacionados con una descortesía mediático-lúdica (Brenes Peña, 2011: 45). La descortesía per se causa un placer intrínseco, que nace de la emoción potencial encerrada en la violencia verbal (Culpeper, 2005: 45), mientras que en el caso de la descortesía indirecta el placer nace del esfuerzo intelectual que supone el reconocimiento de la implicatura y su 
correcta interpretación. De ahí que la transgresión de lo socialmente adecuado -en este caso, las manifestaciones del desacuerdo a través de la descalificación y el insulto o bien de forma indirecta $\mathrm{y}$, por ende, intelectualmente más estimulante- también resulte atractiva y divertida, además de resultar un aliciente para la participación activa para quienes visiten las páginas FB de los festivales.

\section{CONCLUSIONES}

Retomando nuestras reflexiones iniciales sobre el macrogénero página FB de festivales de música, en tanto contexto sociocultural específico en el que se enmarcan nuestras observaciones sobre los fenómenos sociopragmáticos de la (des)cortesía, podemos afirmar que la interacción en el muro de FB no solo es una práctica discursiva, sino también una práctica social. Como tal, se inserta en una red de relaciones de prácticas económicas y culturales en sentido amplio, en el que caben diferentes voces que forman de manera dialéctica creencias, valores y deseos, constituyéndose como una forma de comunicación que transforma radicalmente relaciones e identidades. En la práctica social de la interacción discursiva de FB se construye un proceso activo, interpretativo y colaborador de representación del mundo a la vez que una negociación de relaciones sociales e identitarias (Chouliaraki y Fairclough, 1999: 46; Dann y Liebman Parrinello, 2007). La plantilla y el medio de FB, al proporcionar herramientas para la autopresentación y para la construcción del contexto, son un marco comunicativo que orienta las expectativas convencionalizadas y rutinarias situando la interacción festival/público en un marco amistoso en el que se «habla» (Giese, 1998; Herring, 2011: 1, 2). Creemos que la descortesía, en parte, depende del marco «familiar»y amistoso proporcionado por el género textual de FB, que ofrece una recontextualización del discurso turístico (y comercial) en una red social constituida por pares, unidos por los vínculos de una amistad (real o virtual), por el hecho de hablar de vivencias concretas relacionadas con el turismo musical, por ser «una peña».

La exploración del corpus de referencia nos ha permitido comprobar una serie de dinámicas conflictivas en las que se utilizan distintas estrategias descorteses, entre los que resaltamos los actos de descortesía descarnada y de descortesía encubierta, frutos de una actitud voluntaria del hablante, quien las utiliza para expresar desacuerdo hacia los servicios turísticos o la oferta musical del festival. Podemos afirmar, pues, que la descortesía en nuestro corpus está básicamente orientada a la consecución de objetivos y crea un efecto social positivo entre los amigos, basado en la superposición entre las categorías de autonomía y afiliación (Bravo, 2004b: 30-31); también apreciamos cómo la descortesía por ir en contra de lo establecido capta la atención y puede servir para el entretenimiento y la diversión. A este respecto, creemos que, por mucho que el enfrentamiento directo y «brutal» llame la atención, estamos lejos de la «espectacularización» que se produce en otros medios, como la televisión (Brenes Peña, 2011), en otras redes sociales como Twitter (Díaz Pérez, 2012) o incluso en otras páginas de FB relativas a temas más conflictivos relacionados con la actualidad, la sociedad, la política o las ideologías. Estamos ante la expresión del grado de 
satisfacción con respecto a un servicio turístico-cultural, dentro de un género que forma parte de la comunicación informal en la que el turista se convierte en emisor del discurso turístico para intercambiar opiniones, prodigar consejos y expresar valoraciones. Como afirman González y Sanmartín (2013: 245), bajo las apariencias de un mero intercambio socializador este tipo de perfiles de FB tiene un tenor transaccional evidente: la promoción del destino turístico. Por otro lado, el desacuerdo y la confrontación son formas de interacción bastante frecuentes en nuestra sociedad actual; la pretensión del individuo de mostrarse original y destacar sobre el grupo lo animan a expresar sus opiniones de manera tajante (Brenes Peña, 2011: 12) y hasta ofensiva.

Con todo, es inevitable que en un contexto turístico el insulto y la injuria llamen la atención, pues están encaminados a la descalificación y el desprestigio del festival más que a su buena fama y su promoción. Es evidente que el sector turístico en el entorno 2.0 debe saber gestionar las críticas negativas, por muy contundentes que sean, ya que en la «sociedad de la conversación» quien rige la dinámica de la comunicación es el propio cliente quien produce, consume y promociona el contenido de su información. Para las empresas, y no solo turísticas, hoy en día es imprescindible incorporar la comunicación directa con los clientes, utilizando los sitios web de redes social, cuya eficacia publicitaria compensa el precio de las provocaciones de los usuarios; el hecho de que los amigos compartan opiniones, comenten sus experiencias y declaren sus preferencias de consumo tiene un valor promocional mayor que la expresión de la insatisfacción con respecto al uso de algún servicio. En este espacio de puesta en escena del discurso turístico, el uso de la descortesía dentro de las posibles estrategias argumentativas tiene una fuerte tendencia de captación y es un ritual socio-lingüístico, una estrategia discursiva que sugiere la emoción y fomenta la construcción de una identidad colectiva.

\section{REFERENCIAS BIBLIOGRÁFICAS}

ABELDA MARCO, Marta (2003): «Los actos de refuerzo de la imagen en la cortesía peninsular», Actas del Primer Coloquio del programa Edice, Estocolmo, Universidad de Estocolmo, 298-305.

BERnAl, María (2007): Categorización sociopragmática de la cortesía y de la descortesía, tesis doctoral, Universidad de Estocolmo [en línea]: <http://su.divaportal.org/smash/get/diva2:196989/FULLTEXT01.pdf>. [Consulta: 16/07/2014].

BOYD, danah y ELLISON, Nicole D. (2013): «Sociality through Social Network Sites», en Dutton, William H., ed., The Oxford Handbook of Internet Studies, Oxford, Oxford University Press, 151-172, <http://dx.doi.org/10.1093/oxfordhb/9780199589074.013.0008>.

BRAVo, Diana (2004a): «Panorámica breve acerca del marco teórico y metodológico», en Bravo, Diana y Antonio Briz, eds., Pragmática sociocultural: estudios sobre el discurso de cortesía en español, Barcelona, Ariel Lingüística, 5-11. 
BRAVO, Diana (2004b): «Tensión entre universalidad y relatividad en las teorías de la cortesía», en Bravo, Diana y Antonio Briz, eds., Pragmática sociocultural: estudios sobre el discurso de cortesía en español, Barcelona, Ariel Lingüística, 15-37.

BRENES PEÑA, Esther (2011): Actos del habla disentivos. Identificación y análisis, Sevilla, Alfar.

BRENES PEÑA, Esther (2007) «Los insultos entre los jóvenes: la agresividad verbal como arma para la creación de una identidad grupal», Interlingüística, 17, 200-210.

BRIZ GóMEZ, Antonio (2000): «Turno y alternancia de turno en la conversación» Revista argentina de lingüística, 16, 9-32.

BRIZ GÓMEZ, Antonio (2001 [1998]): El español coloquial en la conversación. Esbozo de pragmagramática, Barcelona, Ariel Lingüística.

Brown, Penelope y Levinson, Stephen (1987 [1978]): Politeness. Some Universals in Language Use, Cambridge, Cambridge University Press.

Bustos Tovar, José Jesús (1999): «El uso del diálogo como mecanismo de información y de opinión», en Garrido Medina, Joaquín, ed., La lengua y los medios de comunicación, vol. 1, Madrid, Servicio de Publicaciones Universidad Complutense de Madrid, 108-117.

CAlsamiglia, Helena y Amparo Tusón (1999): Las cosas del decir, Barcelona, Ariel.

CALVI, Maria Vittoria (2006): Lengua y comunicación en el español del turismo, Madrid, Arco/Libros.

CALvi, Maria Vittoria (2010): «Los géneros discursivos en la lengua del turismo: una propuesta de clasificación», Ibérica, 19 [en línea]: <http://www.aelfe.org/documents/01_19_Calvi.pdf>. [Consulta: 12/07/2010].

CALVI, Maria Vittoria (2011): «Pautas de análisis para los géneros del turismo», en Calvi, Maria Vittoria y Giovanna Mapelli, eds., La lengua del turismo. Géneros discursivos y terminología, Bern, Peter Lang, 19-45.

Charaudeau, Patrick (2009): «Reflexiones para el análisis del discurso populista» Discurso y sociedad, 3 (2), 253-279 [en línea]: $<$ http://www.dissoc.org/ediciones/v03n02/DS3\%282\%29Charaudeau.pdf >. [Consulta: 19/10/2012].

ChIERICHETTI, Luisa (2012): «La promoción del turismo musical: los festivales de música en Facebook», Pasos. Revista de Turismo y Patrimonio Cultural, 10, 4, 49-58 [en línea]: <www.pasosonline.org/Publicados/10412special/PS0412_06.pdf>. [Consulta: 19/2/2014].

ChIERICHETTI, Luisa (2013): «Dinámicas interactivas en la promoción turística: los festivales de música en Facebook», en Chierichetti, Luisa y Giovanni Garofalo, eds., Discurso profesional y lingüistica de corpus. Perspectivas de investigación, Bérgamo, CELSB, 73-99 [en línea]: 〈http://hdl.handle.net/10446/29361>. [Consulta: 19/2/2014].

ChouliaraKi, Lilie y Norman FAIRCLOUGH (1999): Discourse in Late Modernity. Rethinking Critical Discourse Analysis, Edimburgo, Edinburgh University Press.

CULPEPER, Jonathan (1996): «Towards an anatomy of impoliteness», Journal of Pragmatics, 25, 349-367. 
CULPEPER, Jonathan (2005): «Impoliteness and Entertainment in the Television Quiz Show: The Weakest Link», Journal of Politeness Research, 1, 35-72.

CULPEPER, Jonathan, Derek BousFIELD y Anne WiCHMANN (2003): «Impoliteness revisited: with special reference to dynamic and prosodic aspects», Journal of Pragmatics, 35, 1545-1579.

DANN, Graham M. S. y Giuli LiEBMAN PARRINELlo (2007): «From Travelogue to Travelblog: (Re)-negotiating Tourist Identity», Acta Turistica, 19/1, 7-29.

DÍAZ PÉREZ, Juan Carlos (2012): Pragmalingüística del disfemismo y la descortesía, tesis doctoral, Universidad Carlos III de Madrid [en línea]: $<$ E_archivo.uc3m.es/bitstream/10016/15682/1/Tesis_Doctoral_Juan_Carlos_Diaz_Pere z_UC3M_2012.pdf>. [Consulta: 19/10/2012].

ESCANDELl VIDAL, María Victoria (2006): Introducción a la pragmática, Barcelona, Ariel.

GARCÉS-CONEJOS BlitVICH, Pilar (2010): «A genre approach to the study of impoliteness», International Review of Pragmatics, 2, 1: 46-94.

GARCÉS-CONEJOS BLITVICH, Pilar (2013): «El modelo del género y la des/cortesía clasificatoria en las evaluaciones de Sálvame por parte de la audiencia», en Fuentes, Catalina, ed., (Des)cortesía para el espectáculo; Estudios de pragmática variacionista, Madrid, Arco/Libros.

GARCÍA IZQUIERDO, Isabel y Esther Monzó NEBOT (2003): «Una enciclopedia para traductores. Los géneros de especialidad como herramienta privilegiada del traductor profesional», en Muñoz Martín, Ricardo, ed., I AIETI. Actas del I Congreso Internacional de la Asociación Ibérica de Estudios de Traducción e Interpretación, 1. Granada, 12-14 de febrero de 2003, Granada, AIETI, 83-97 [en línea]: $\langle$ http://www.aieti.eu/pubs/actas I/AIETI_1_IGI_EMN_Enciclopedia.pdf>. [Consulta: 19/10/2012]. Mulino.

GIDDENS, Anthony (1994 [1990]): Le conseguenze della modernità, Bologna, Il

GIESE, Mark (1998): «Self Without Body; Textual Self-Representation in an Electronic Community» First Monday 3, 4, 6, abril 1998, <http://dx.doi.org/10.5210/fm.v3i4.587>.

GONZÁLEZ, Virginia y Julia SANMARTín (2013): «Facebook y discurso turístico: tenor funcional y estrategias pragmalingüísticas en turismo Madrid y turismo Sevilla», en Cabedo Nebod, Adrián, Manuel José Aguilar Ruiz y Elena López-Navarro Vidal, eds., Estudios de lingüística. Investigaciones, propuestas y aplicaciones, Valencia, Universitat de València, 243-252 [en línea]: $\langle$ http://www.uv.es/canea/archivos/Estudios_linguistica_2013.pdf $>. \quad$ [Consulta: 19/2/2014].

GRAPPI, Silvia y Fabrizio MONTANARI (2011): «The role of social identification and hedonism in affecting tourist re-patronizing behaviours: The case of an Italian festival», Tourism $\quad$ Management, $\quad 32, \quad 1128-1140$, <http://dx.doi.org/10.1016/j.tourman.2010.10.001>. 
HERNÁNDEZ FlORES, Nieves (2004): «La cortesía como búsqueda del equilibrio de la imagen social», en Bravo, Diana y Antonio Briz, eds., Pragmática sociocultural: estudios sobre el discurso de cortesía en español, Barcelona, Ariel Lingüística, 95-134.

HERRERO, Gemma (1996): «La importancia del concepto de enunciado en la investigación del español coloquial: a propósito de enunciados suspendidos», en Briz Gómez, Antonio, ed., Pragmática y gramática del español hablado: actas del II simposio sobre análisis del discurso oral, Zaragoza, Pórtico, 109-126.

HERRING, Susan C. (2004): «Computer-Mediated Discourse Analysis: An Approach to Researching Online Behavior», en Barab, Sasha A., Rob Kling, Rob y James H. Gray, eds., Designing for Virtual Communities in the Service of Learning, Nueva York, Cambridge University Press, 338-376.

HERRING, Susan C. (2011): «Computer-Mediated Conversation: Introduction and Overview», Language@Internet, $8, \quad$ artículo 2 [en línea]: $<$ http://www.languageatinternet.org/articles/2011/Herring>. [Consulta: 13/11/2012].

Kerbrat-Orecchioni, Catherine (1986): La enunciación. De la subjetividad en el lenguaje, Buenos Aires, Hachette.

Kerbrat-Orecchioni, Catherine (2004): «Introducing Polylogue», Journal of Pragmatics, 36, 1-24, <http://dx.doi.org/10.1016/s0378-2166(03)00034-1〉.

ILIE, Cornelia (2001): «Unparliamentary Language: Insults as Cognitive Forms of Ideological Confrontation», en Dirven, René, Roslyn Frank y Cornelia Ilie, eds., Language and Ideology. Volume II: Descriptive Cognitive Approaches, Ámsterdam/Filadelfia, Johns Benjamins, 235-263, <http://dx.doi.org/10.1075/cilt.205.14ili>.

LAKOFF, Robin T. (1973): «The logic of politeness, or minding your p's and q's», Proceedings of the Ninth Regional Meeting of the Chicago Linguistics Society, 9, 292-305.

LANDONE, Elena (2009): Los marcadores del discurso y cortesía verbal en español, Bern, Peter Lang.

LEECH, Geoffrey (1983): Principles of pragmatics, Londres, Longman.

NiCOLÁs OJEDA, Miguel Ángel (2012): «Consideraciones sobre comunicación publicitaria y redes sociales: usuarios, características, estrategias y planificación», en Nicolás Ojeda, Miguel Ángel y María del Mar Grandío Pérez, coords., Estrategias de comunicación en redes sociales. Usuarios, aplicaciones y contenidos, Barcelona, Gedisa, 13-30.

NiCOLÁs OJEDA, Miguel Ángel (2013): «La implicación del usuario en la producción publicitaria. Una reflexión sobre la publicidad espontánea generada por los usuarios/consumidores», $\quad$ Icono, $14, \quad 11, \quad 303-317$, <http://dx.doi.org/10.7195/ri14.v11i1.204>.

PARKS, Malcolm R. (2010): «Who are Facebook Friends?». Paper presented at the International Communication Association annual meeting, Singapore.

SANMARTín SÁEZ, Julia (2007): El chat. La conversación tecnológica, Madrid, Arco/Libros.

UPADHYAY, Shiv R. (2010): «Identity and impoliteness in computer-mediated reader responses», Journal of Politeness Research, 6, 105-127, <http://dx.doi.org/10.1515/jplr.2010.006>. 
ZIMMERMAN, Klaus (2003): «Constitución de la identidad y anticortesía verbal entre jóvenes masculinos hablantes de español», Actas del Primer Coloquio del programa Edice, Estocolmo, Universidad de Estocolmo, 47-59. 